\title{
Coupling and propagation of normal and dysrhythmic gastric slow waves during acute hyperglycaemia in healthy humans
}

\author{
R. COLESKI \& W. L. HASLER \\ Division of Gastroenterology, University of Michigan Health System, Ann Arbor, MI, USA
}

\begin{abstract}
Acute hyperglycaemia in healthy humans disrupts slow wave rhythm similar to that observed in diabetic gastropathy, but hyperglycaemic effects on regional dysrhythmias, power, coupling and propagation are unexplored. Using mucosal mapping, we aimed to demonstrate that hyperglycaemia elicits region-specific tachygastria and evokes slow wave uncoupling between adjacent regions. Catheters with bipolar electrodes were affixed 10.5, 6 and $2 \mathrm{~cm}$ from the pylorus during endoscopy with midazolam in 10 healthy humans. Recordings were obtained for $1 \mathrm{~h}$ under basal conditions and for $1 \mathrm{~h}$ with hyperglycaemic clamping to $250 \mathrm{mg} d L^{-1}$. In basal recordings, proximal and distal slow wave frequencies were similar $[2.91 \pm 0.05$ vs $2.81 \pm 0.09$ cycles per minute $(\mathrm{cpm})]$. Tachygastria $(>3.6 \mathrm{cpm})$ was present $1.7 \pm 1.1 \%$ of the time proximally and $3.3 \pm 1.8 \%$ distally and localized to one lead $67 \%$ of the time. Proximal to distal gradients in signal power and power variability were observed. Coupling between adjacent sites was $78 \pm 2 \%$ with propagation velocities of $1.3 \pm 0.1 \mathrm{~cm} \mathrm{~s}^{-1} .2 \pm 1 \%$ of segments showed $>50 \%$ uncoupling. Hyperglycaemic clamping increased mean proximal $(3.18 \pm 0.11 \mathrm{cpm})$ and distal $(3.50 \pm 0.12 \mathrm{cpm})$ frequencies and proximal $(15 \pm 6 \%)$ and distal $(32 \pm 9 \%)$ tachygastria (all $P<0.01)$ that localized to one lead $80 \%$ of the time. During periods of normal frequency, coupling decreased proximally $(54 \pm 6 \%)$ and distally $(47 \pm 4 \%)(P<0.01) .55 \pm 8 \%$ of segments showed $>50 \%$ uncoupling $(P<0.01)$. In conclusion, gastric slow waves show stable, highly coupled rhythms under basal conditions. Hyperglyca-
\end{abstract}

Address for correspondence

William L. Hasler, MD, 3912 Taubman Center, Box 0362, Ann Arbor, MI 48109, USA.

Tel: +1 734936 4780; fax: +1 734936 7392;

e-mail: whasler@umich.edu

Received: 26 June 2008

Accepted for publication: 5 October 2008 emia elicits isolated tachygastrias and uncoupling of normal slow waves that are most prominent distally. These findings provide a foundation for studying slow wave conduction defects in diabetic gastropathy.

Keywords diabetes mellitus, gastric electrophy siology, gastropathy, hyperglycaemia.

Several factors are proposed to contribute to the pathogenesis of diabetic gastropathy, including impaired gastric emptying and motor abnormalities involving the proximal and distal stomach, pylorus, and small intestine. ${ }^{1-5}$ Antropyloric motor activity is regulated by a cyclic myoelectric phenomenon, the slow wave, which controls the maximal frequency and direction of phasic pressure waves. ${ }^{6}$ Prominent gastric myoelectric disruptions are demonstrable in subsets of patients with diabetic gastropathy and are postulated to participate in symptom genesis. ${ }^{7,8}$

Gastric dysrhythmic activity in diabetic gastropathy relates strongly to the degree of glycaemic control. Maintenance of euglycaemia nearly abolishes tachygastrias that are observed during periods of elevated glucose in diabetics. ${ }^{9}$ Furthermore in addition to disrupting normal gastric motor activity, acute hyperglycaemia elicits tachygastrias in healthy humans which are associated with loss of distal gastric phasic contractions. ${ }^{10}$ These findings indicate a prominent role for metabolic factors rather than fixed neuromuscular defects in the myoelectric disturbances of diabetes. Electrogastrography has been employed to document these abnormalities; this technique acquires gastric electrical signals from cutaneous electrodes affixed to the abdominal wall overlying the stomach. ${ }^{11}$ Although this method integrates and sums myoelectrical events occurring in the stomach, it cannot quantify regional differences in slow wave rhythm and power, coupling between adjacent gastric regions or slow wave propagation characteristics. 
Our laboratory recently validated a method to map localized variations in slow wave activity using an endoscopically directed bipolar electrode system. In healthy humans, this technique characterized regional myoelectric effects of two distinct gastric dysrhythmic stimuli - intravenous glucagon and acute hyperglycaemia. ${ }^{12,13}$ However, this recording system consisted of only a single bipolar electrode that was pressed upon but not affixed to the gastric mucosa. Its limitations included the ability to record signals only for short periods of time, the requirement that the endoscope remain in the stomach during recording, and an inability to record simultaneous myoelectric activity from two or more sites. Thus, this method provided limited information regarding intermittent slow wave disruption and coupling and could not assess propagation direction or velocity.

In the present investigation, we employed a modified recording system that was comprised of three bipolar electrodes that could be reliably affixed to the gastric mucosa for several hours after being placed by upper endoscopy. Using this method, we aimed to test the hypotheses that acute hyperglycaemia (i) elicits slow wave dysrhythmias that vary by gastric region and (ii) evokes slow wave uncoupling between adjacent regions. Simultaneous mucosal recordings were obtained from the gastric body and proximal and distal gastric antrum in healthy human volunteers under basal conditions and during hyperglycaemic clamping to $250 \mathrm{mg} \mathrm{dL}^{-1}$. The effects of hyperglycaemia on several slow wave parameters, including dominant frequency, per cent time in tachygastria, signal power, power variability, per cent coupling between adjacent sites, per cent of recording segments with predominant uncoupling, and conduction direction and velocity were assessed. Through these investigations, we hoped to form the foundation for studying slow wave disturbances in conditions with myoelectric disruption such as diabetic gastropathy.

\section{MATERIALS AND METHODS}

\section{Human subjects}

Ten healthy volunteers (seven men and three women, mean age 26 years - range 19-43 years) were recruited by campus wide advertisement. Volunteers had no prior gastrointestinal disease or symptoms, had undergone no surgery involving the gastrointestinal tract, and were on no medications that alter gastrointestinal motor or myoelectrical activity. The study was approved by the Institutional Review Board at the
University of Michigan Hospital. All subjects provided written informed consent.

\section{Experimental design}

After overnight fasting, volunteers underwent multichannel mucosal slow wave mapping in the General Clinical Research Center at the University of Michigan Hospital under two test conditions during a single session: (i) basal euglycaemic conditions and (ii) hyperglycaemic clamping with intravenous infusion of $20 \%$ dextrose to stable plasma glucose levels of $250 \mathrm{mg} \mathrm{dL}^{-1}$.

Multichannel recording electrode placement Catheters were inserted into antecubital veins in each arm for administration of conscious sedation for performance of upper endoscopy, measurement of blood glucose, and infusion of $20 \%$ dextrose. Patency of the intravenous lines was maintained with heparin flushlock solution (100 USP units per millilitre). Blood pressure, pulse and pulse oximetry were continuously monitored (Dash 3000; GE Marquette Medical Systems, Milwaukee, WI, USA) during and for $1 \mathrm{~h}$ after endoscopies. Prior to endoscopy, the pharynx was sprayed with topical benzocaine (Buetlich LP Pharmaceuticals, Waukegan, IL, USA) and conscious sedation was achieved using intravenous midazolam $(4-10 \mathrm{mg})(\mathrm{FH}$ Faulding \& Co., Ltd., Elizabeth, NJ, USA). A suture on the tip of a three-channel bipolar recording electrode constructed from three modified BICAP haemostasis probes (Model BP-7300A, Circon Corp., Racine, WI, USA) was grasped by biopsy forceps passed through an upper endoscope (Model EG-2731, Pentax Precision Instrument Corp., Orangeburg, NY, USA). The upper endoscope was introduced orally with the multichannel electrode system 'aside the scope' into the distal stomach under direct visualization. The three bipolar probes were positioned 2, 6 and $10.5 \mathrm{~cm}$ proximal to the pylorus along the greater curve of the stomach (Fig. 1). The biopsy forceps was withdrawn and preloaded endoclip delivery catheters (Quickclip HX-200L-135, Olympus America Inc. Two Corporate Center Drive, Melville, NY, USA) were passed through the internal channel of the endoscope. Six endoclips were deployed to affix the recording electrode to the gastric mucosa two clips for each bipolar probe. The endoscope was withdrawn and the electrodes were connected via direct nystagmus couplers (Model 9859, SensorMedic, Anaheim, CA, USA) to a chart recorder for continuous display of electrical activity. Time constants were set at $10 \mathrm{~s}$ and high-frequency cutoffs were set at $0.3 \mathrm{~Hz}$ to minimize interference from non-gastric signals. 


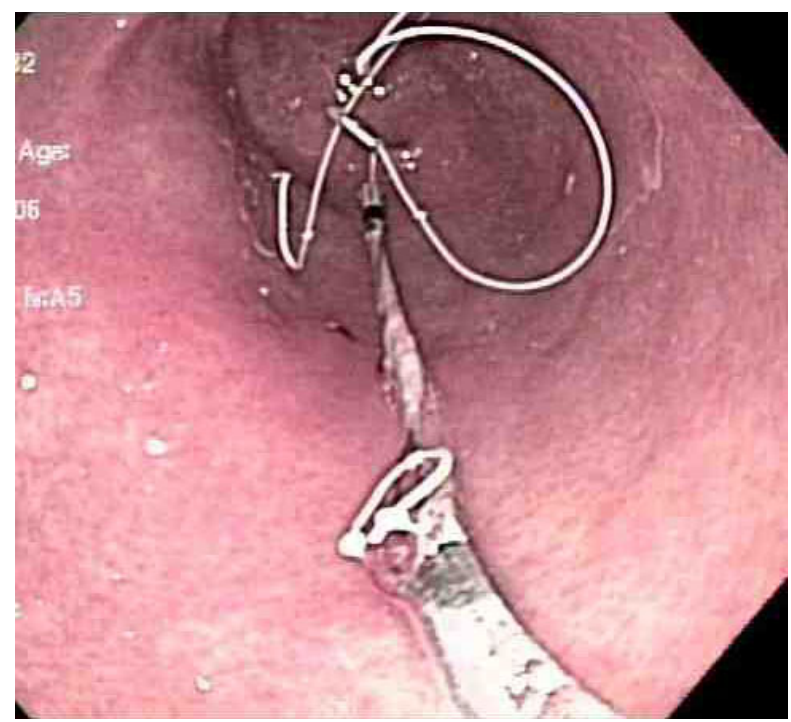

Figure 1 This photograph shows the endoscopic placement of the multichannel mucosal recording electrode system with the most distal probe affixed to the gastric mucosa with an endoclip $2 \mathrm{~cm}$ proximal to the pylorus and positioning of the middle probe $6 \mathrm{~cm}$ proximal to the pylorus prior to mucosal clipping.

Mucosal slow wave recording protocol After a $30 \mathrm{~min}$ equilibration period, an $1 \mathrm{~h}$ baseline multichannel recording was performed under euglycaemic conditions. Blood glucose levels were measured at the beginning and end of the baseline period using a portable glucose analyser (ACCU-CHEK; Roche Diagnostic Corporation, Indianapolis, IN, USA) to confirm euglycaemia. Hyperglycaemic clamping was initiated employing a validated method with monitoring of glucose levels every 5 min. ${ }^{14}$ A 15 min priming infusion of $20 \%$ dextrose was delivered, then infusions were lowered to maintenance rates which were adjusted as indicated by results of glucose measurements. Multichannel recording was performed for $1 \mathrm{~h}$ during hyperglycaemic clamping. In general, the desired blood glucose target of $250 \mathrm{mg} \mathrm{dL}^{-1}$ was achieved within $15 \mathrm{~min}$ and was maintained within $\pm 25 \mathrm{mg} \mathrm{dL}^{-1}$ for the duration of the recording.

Upon completion of the recordings, the dextrose infusion was reduced over $15 \mathrm{~min}$. Glucose values were obtained every $10 \mathrm{~min}$ to ensure rebound hypoglycaemia did not occur. The pharynx was resprayed with benzocaine and additional midazolam (4-9 mg) was administered to reachieve conscious sedation. The endoscope was reintroduced and the endoclips were detached from the mucosa using foreign body retrieval forceps (Model: FG-6U, Olympus America Inc. Two Corporate Center Drive). The endoscope and recording electrode were withdrawn orally and blood pressure, pulse, and pulse oximetry were monitored for at least $1 \mathrm{~h}$. Subjects were then fed a mixed meal and discharged under the supervision of a designated driver.

Signal analysis Multichannel mucosal recording data were transferred to a personal computer $(4 \mathrm{DX} 2-66 \mathrm{~V}$, Gateway 2000, North Sioux City, ND, USA) via an analogue-to-digital converter (DAS-16, Metrabyte, Taunton, MA, USA). Signals were digitized at $4 \mathrm{~Hz}$ and filtered above 15 cycles per minute $(\mathrm{cpm})$ and below $0.5 \mathrm{cpm}$ to remove high- and low-frequency noise. Power spectral analyses were performed across the frequency range from 1 to $9 \mathrm{cpm}$ on $256 \mathrm{~s}$ segments of recording with $76 \%$ overlap. The frequency range $\geq 2.4$ and $\leq 3.6 \mathrm{cpm}$ was defined to represent normal. The frequency range $>3.6$ and $<9 \mathrm{cpm}$ was defined as tachygastria, and the frequency range from $\geq 1$ and $<2.4 \mathrm{cpm}$ was defined as bradygastria. Frequencies $<1 \mathrm{cpm}$ most often resulted from a mildly unstable baseline and did not represent gastric myoelectric cycling - this frequency band was not analysed. Identification of artefactual signals not originating from the stomach was made visually by an investigator blinded to the test condition. Artefacts were readily identifiable as abrupt signal deflections that went off scale, as described for electrogastrography. ${ }^{15}$ In some cases, such artefacts resulted from abrupt subject movements as with coughing. These artefacts most often were observable in all the three mucosal recordings. In other instances, no obvious subject movement was evident and transient loss of electrode contact with the mucosa was the likely cause of signal loss. Most such artefacts were observable only in a single recording. Analyses performed on recording segments with signal artefact were not included in final data calculations.

Several parameters were calculated for each site during recordings under basal and hyperglycaemic conditions. Mean dominant frequencies were determined for each entire basal and hyperglycaemia period. Percentages of $256 \mathrm{~s}$ recording segments with dominant frequencies in the normal, tachygastric and bradygastric ranges were calculated. Visible tachygastrias lasting $\geq 2 \mathrm{~min}$ were identified visually from recording segments with dominant frequencies $>3.6 \mathrm{cpm}$ and quantified and were localized to one or more leads. Power in the dominant frequency range for each time segment was measured at each site. Temporal variability of the power for a given subject was determined by calculating the standard deviation of the power of each $256 \mathrm{~s}$ recording segment. Both power parameters were plotted as a fraction of the value measured from the proximal lead $10.5 \mathrm{~cm}$ from the 
pylorus. The per cent slow wave coupling between adjacent recording sites was calculated only during periods of normal slow wave frequency with antegrade propagation and was defined as deviation of $<25 \%$ of the slow wave cycle from parallelism of lines connecting the peaks from adjacent recording channels as previously described. ${ }^{16}$ The percentages of $256 \mathrm{~s}$ recording segments with $<50 \%$ coupling were determined using a similar definition. This parameter was arbitrarily chosen with the rationale that a loss of coupling of more than half of slow waves in adjacent gastric regions would potentially decrease propagated motor activity $50 \%$ or more in these regions. Visual comparisons of recordings from the three probes were performed to determine the direction of slow wave propagation. Propagation velocities were calculated by dividing distances between adjacent bipolar probes by the time difference between peaks of propagated slow waves.

\section{Statistical analyses}

Results were expressed as mean \pm SEM. Single factor ANOVA was performed to determine if there were significant differences in dominant frequency, percentages of recording time in each frequency range, signal power and power variability between the three recording sites for each test condition. The Tukey Honestly Significant Differences method was employed to test if absolute differences between any two sample mean values were significantly different. Paired Student's $t$ testing was performed to assess differences in \% coupling, \% of recording segments with $<50 \%$ coupling, and propagation velocities between the proximal-middle and middle-distal pairs of recording sites for each test condition. Paired Student's testing was performed for comparisons of slow wave parameters between the basal recordings and recordings obtained during acute hyperglycaemia. A $P$-value of $<0.05$ defined statistical significance.

\section{RESULTS}

All subjects completed mucosal recordings under basal and hyperglycaemic clamping conditions. Mucosal recordings were analysed for several slow wave parameters. Because some degree of sedation was present during mucosal recordings, symptom assessments could not reliably be made. Sample multichannel tracings are shown in Fig. 2. Under basal conditions, regular slow wave recordings were obtained that exhibited a period of approximately $20 \mathrm{~s}$ (Fig. 2A). Propagation was antegrade and cycling was highly coupled between leads under basal conditions (dashed lines). Conduction velocities in this individual averaged $0.9 \mathrm{~cm} \mathrm{~s}^{-1}$. During hyperglycaemic clamping to $250 \mathrm{mg} \mathrm{dL}^{-1}$, slow wave activity cycling was disrupted in all three leads (Fig. 2B). The distal lead in particular exhibited a chaotic pattern with many recording segments exhibiting decreases in period to $7.5-15 \mathrm{~s}$ and increases in mean dominant frequency to $4-8 \mathrm{cpm}$. Coordinated propagation could not be identified for extended segments of time in recordings from this individual.

\section{Hyperglycaemic effects on gastric slow wave frequency}

The mean recording time analysed under basal conditions was $57 \pm 2$ min per subject. The remaining time could not be analysed due to artefact (respiratory or gastric contractile) or loss of electrode contact with the mucosa. The mean recording time with blood glucose values within $\pm 10 \%$ of $250 \mathrm{mg} \mathrm{dL}^{-1}$ during hyperglycaemic clamping was $41 \pm 1$ min per subject. Signals acquired prior to achieving the target glycaemic range were not analysed.

Slow wave frequency parameters were compared under basal conditions and during hyperglycaemic clamping to $250 \mathrm{mg} \mathrm{dL}^{-1}$. Under basal conditions, dominant frequencies were stable in the proximal $(2.91 \pm 0.05 \mathrm{cpm})$, middle $(2.91 \pm 0.04 \mathrm{cpm})$, and distal $(2.81 \pm 0.09 \mathrm{cpm})$ leads (Fig. 3A). Tachygastric activity was infrequent and occurred similarly in the three leads $(P=$ ns), being identified during $2 \pm 1 \%$ of recording time in the proximal lead, $3 \pm 2 \%$ of the time in the middle lead and $3 \pm 2 \%$ of recording time distally (Fig. 3B). Six discrete tachygastric bursts were observed lasting $\geq 2 \mathrm{~min}$; four were localized to one recording lead (Table 1). Hyperglycaemic clamping elicited disruptions in normal slow wave cycling that were evident at blood glucoses above $200 \mathrm{mg} \mathrm{dL}^{-1}$. Dominant frequencies during hyperglycaemia were increased in the proximal $(3.18 \pm 0.11 \mathrm{cpm}, P=0.01)$ and distal $(3.50 \pm 0.12 \mathrm{cpm}, P<0.01)$ leads and showed trends to increases in the middle $(3.18 \pm 0.15, P=0.12)$ lead (Fig. 3A). Dominant frequencies distally were greater than those proximally during hyperglycaemia $(P=0.03)$. Tachygastric activity showed trends to increases in the proximal lead to $15 \pm 6 \%(P=0.07)$ and in the middle lead to $17 \pm 7 \%(P=0.09)$, and significant increases in the distal lead to $32 \pm 9 \%$ $(P<0.01)$ (Fig. 3B). Fifty discrete tachygastric episodes $\geq 2$ min were recorded during hyperglycaemia (Table 1). Forty-two $(80 \%)$ were noted in a single lead. Isolated tachygastrias were more common distally (23 episodes) 

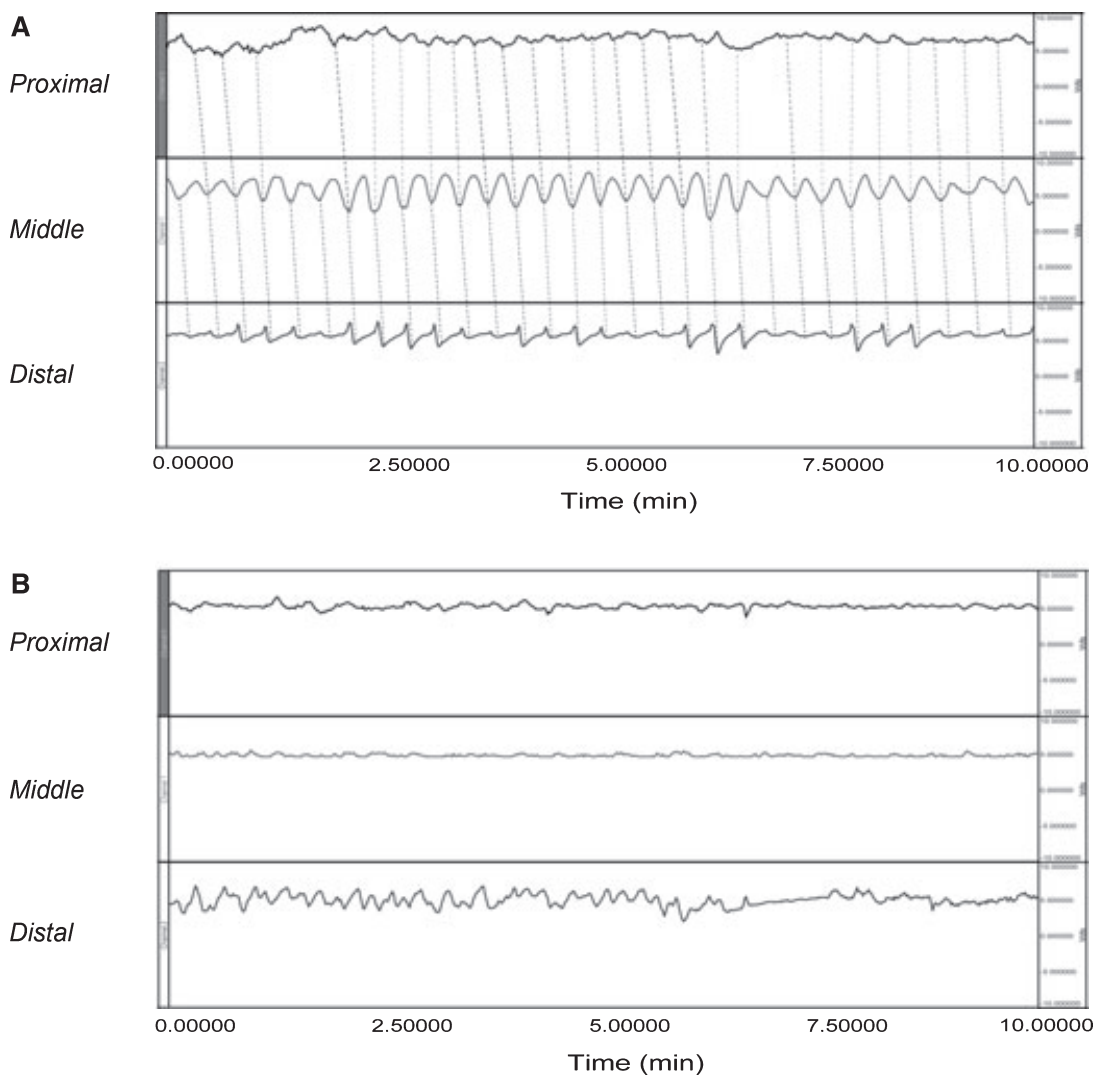

Figure 2 Representative multichannel mucosal slow wave recordings are shown from a healthy volunteer. Under basal conditions (A), slow waves from the proximal (top), middle and distal (bottom) leads exhibit a regular oscillation with a period of approximately $20 \mathrm{~s}$. The dashed lines show the close coupling between adjacent leads. With hyperglycaemic clamping to $250 \mathrm{mg} \mathrm{dL}^{-1}$, there is loss of the stable rhythm that is most prominent in the distal lead (B). In this recording, the regular rhythm is replaced by a high frequency, chaotic waveform with a mean period of approximately $10 \mathrm{~s}$ and poor coupling.
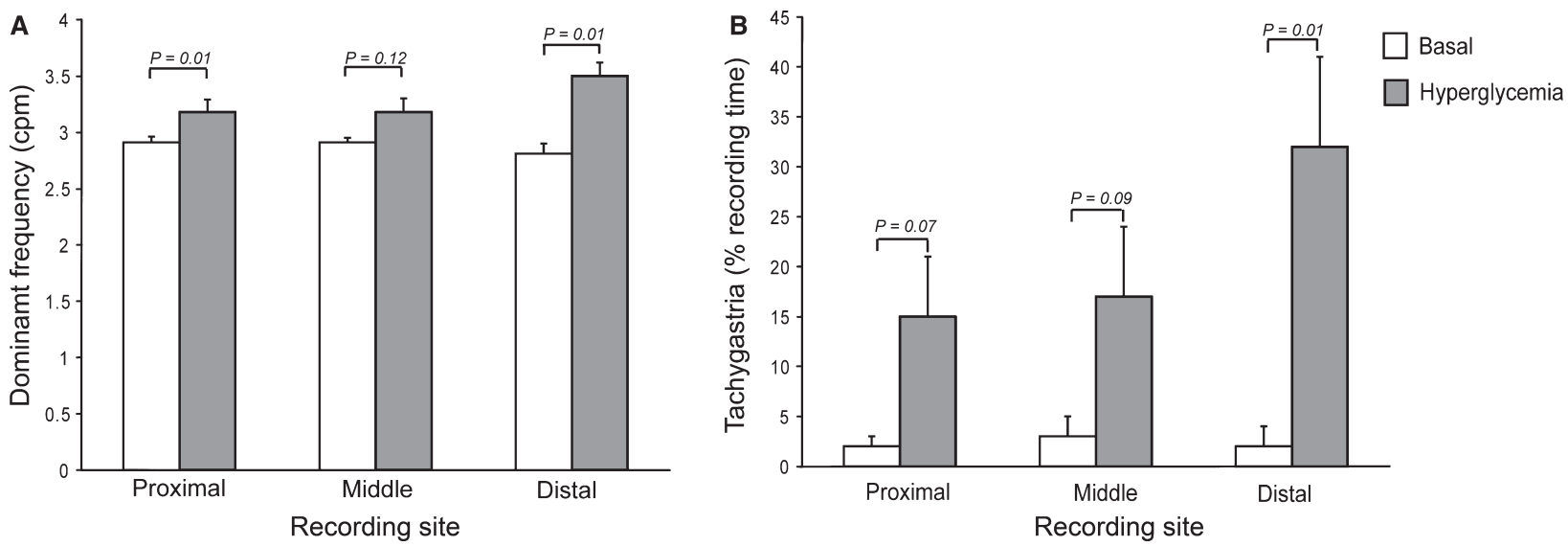

Figure 3 These graphs plot the mean dominant slow wave frequencies for each recording site (A) and the percentages of recording time in tachygastria (B) under the two test conditions. Under basal conditions, dominant frequencies are similar across all leads and range from 2.81 to 2.91 cycles per minute $(\mathrm{cpm})$ (A). With hyperglycaemic clamping to $250 \mathrm{mg} \mathrm{dL}^{-1}$, there are significant increases in dominant frequency in the proximal and distal leads and trends to increases in the middle lead. The dominant frequency increase distally is greater than the increase in the proximal and middle leads. Under basal conditions, tachygastric activity is infrequent in all recording leads (B). With hyperglycaemic clamping to $250 \mathrm{mg} \mathrm{dL} \mathrm{L}^{-1}$, there are trends to increased tachygastria in the proximal and middle leads and significant increases in tachygastria in the distal lead to $32 \%$ of recording time. 
Table 1 Characteristics of discrete tachygastric episodes

\begin{tabular}{llll}
\hline Test condition & $\begin{array}{l}\text { Number of } \\
\text { tachygastric } \\
\text { episodes }\end{array}$ & $\begin{array}{l}\text { Number }(\%) \\
\text { of isolated } \\
\text { tachygastrias }\end{array}$ & $\begin{array}{l}\text { Distribution } \\
\text { of isolated } \\
\text { tachygastrias }\end{array}$ \\
\hline Basal & 6 & $4(67 \%)$ & $\begin{array}{l}\text { Proximal } 1(25 \%) \\
\text { Middle } 1(25 \%)\end{array}$ \\
$\begin{array}{l}\text { Hyperglycaemic } \\
\text { clamping }\end{array}$ & 50 & $42(80 \%)$ & $\begin{array}{l}\text { Distal } 2(50 \%) \\
\text { Proximal } 8(19 \%) \\
\text { Middle } 11(26 \%)\end{array}$ \\
Distal $23(55 \%)$
\end{tabular}

than proximally (eight episodes) $(P<0.01)$. Hyperglycaemia did not increase times in bradygastria $15 \pm 2 \%$ under basal conditions vs $6 \pm 3 \%$ during hyperglycaemia, $P=0.60$ ).

\section{Hyperglycaemic effects on gastric slow wave power}

Signal power and temporal variability of power were analysed under basal and hyperglycaemic conditions. Under basal conditions, signal power exhibited a proximal to distal gradient (Fig. 4A). Power in the distal lead was $2.8 \pm 0.5$-fold that of the power in the proximal lead $(P<0.01)$. Likewise, power variability distally was $2.5 \pm 0.5$-fold that of measured proximally $(P=0.02) \quad$ (Fig. 4B). Hyperglycaemic clamping to $250 \mathrm{mg} \mathrm{dL}^{-1}$ did not affect signal power compared with basal conditions $(P=0.87)$, but showed a trend to reducing the proximal to distal power gradient $(1.9 \pm 0.4, P=0.09)$ (Fig. 4A). Similarly, hyperglycaemia did not alter the temporal variability of slow wave power compared with basal conditions $(P=0.58)$ (Fig. 4B). Hyperglycaemia also had no effect on the proximal to distal gradient in the temporal variability of power $(2.3 \pm 0.9, P=0.69)$.

\section{Hyperglycaemic effects on gastric slow wave propagation parameters}

Slow wave coupling between adjacent sites and propagation velocities were compared under basal conditions and during hyperglycaemic clamping to $250 \mathrm{mg} \mathrm{dL}^{-1}$. During basal recording periods, conduction was antegrade in direction. Coupling between the proximal and middle leads was $76 \pm 3 \%$, while coupling between the middle and distal leads was $80 \pm 2 \%$ (Fig. 5A). The per cent of recording segments with $<50 \%$ coupling was $1 \pm 1 \%$ proximally and $2 \pm 2 \%$ distally (Fig. 5B). Propagation velocities between the proximal and middle leads were $1.2 \pm 0.1 \mathrm{~cm} \mathrm{~s}^{-1}$, while velocities between the middle and distal leads were $1.3 \pm 0.2 \mathrm{~cm} \mathrm{~s}^{-1}$ (Fig. 6). Hyperglycaemic clamping
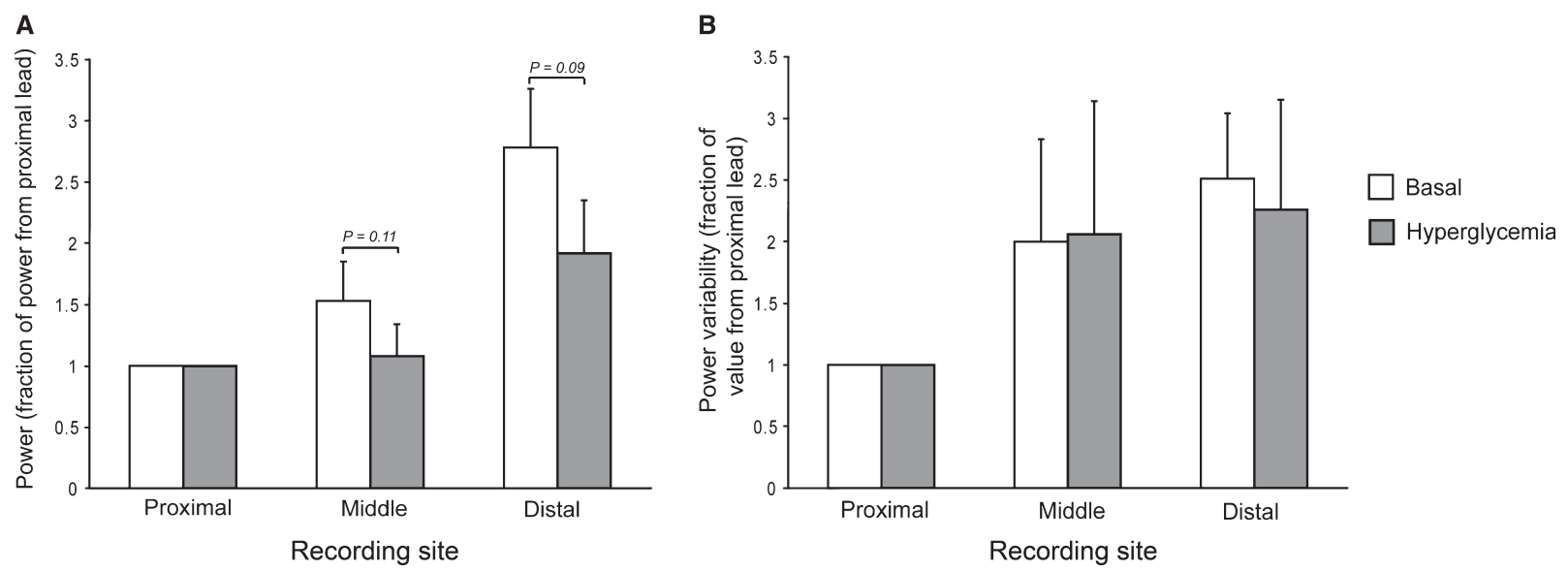

Figure 4 These graphs plot the slow wave signal power values (A) and the temporal variabilities in slow wave power (B) as fractions of the power at the proximal recording lead for each site under the two test conditions. There is a significant increasing proximal to distal power gradient under basal conditions (A). There is a trend to a decrease in the proximal to distal power gradient during hyperglycaemic clamping to $250 \mathrm{mg} \mathrm{dL}^{-1}$. Under both basal conditions and during hyperglycaemic clamping to $250 \mathrm{mg} \mathrm{dL}^{-1}$, there is greater power variability in the middle and distal leads than in the proximal lead (B). However, there are no differences in power variability between the two test conditions. 

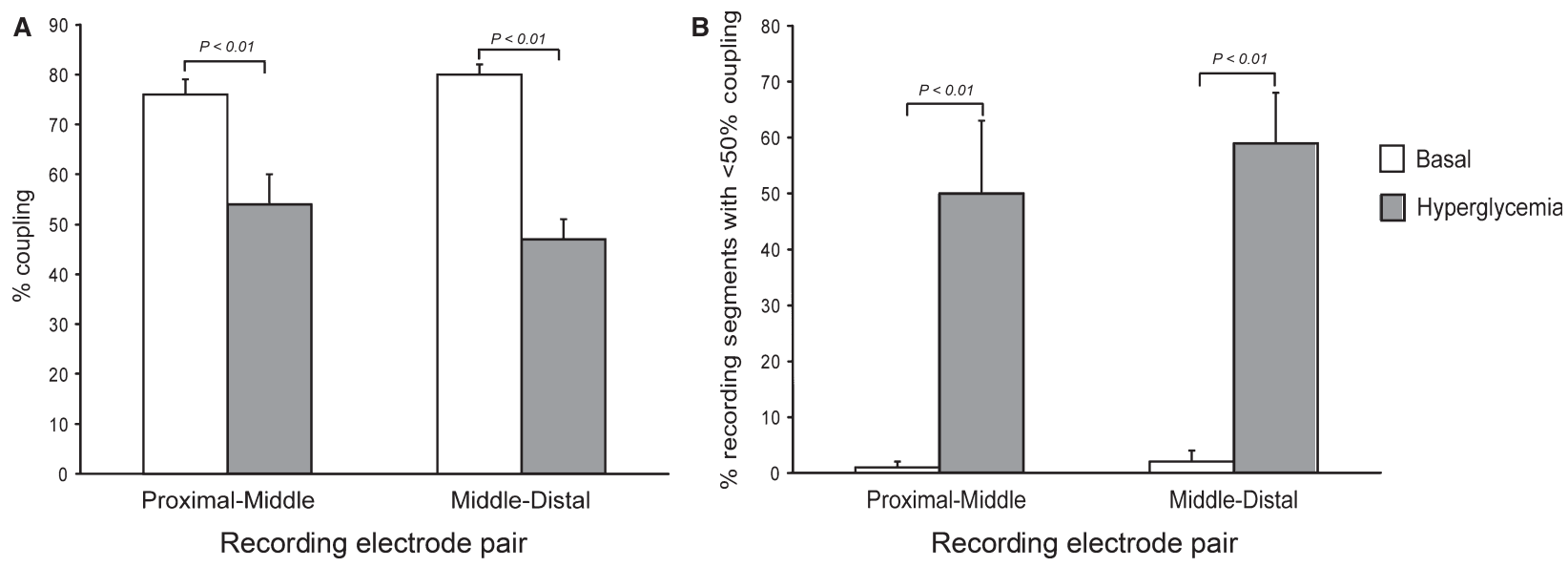

Figure 5 These graphs plot the percentages of coupling of slow waves of normal frequency (A) and the percentages of recording segments with $<50 \%$ coupling (B) from the proximal to the middle recording site and from the middle to the distal site under the two test conditions. Under basal conditions, coupling between adjacent leads is tight ranging from $74 \%$ proximally to $80 \%$ distally (A). Hyperglycaemic clamping to $250 \mathrm{mg} \mathrm{dL}^{-1}$ promotes a loss of coupling between both electrode pairs to $54-47 \%$ respectively. There are no significant differences in proximal vs distal coupling. Very few recording segments exhibit $<50 \%$ coupling under basal conditions (B). With hyperglycaemic clamping to $250 \mathrm{mg} \mathrm{dL}^{-1}$, there is a significant increase in this parameter between both electrode pairs. There are no significant differences in the percentages of recording segments with $<50 \%$ coupling in the proximal vs distal electrode pairs.

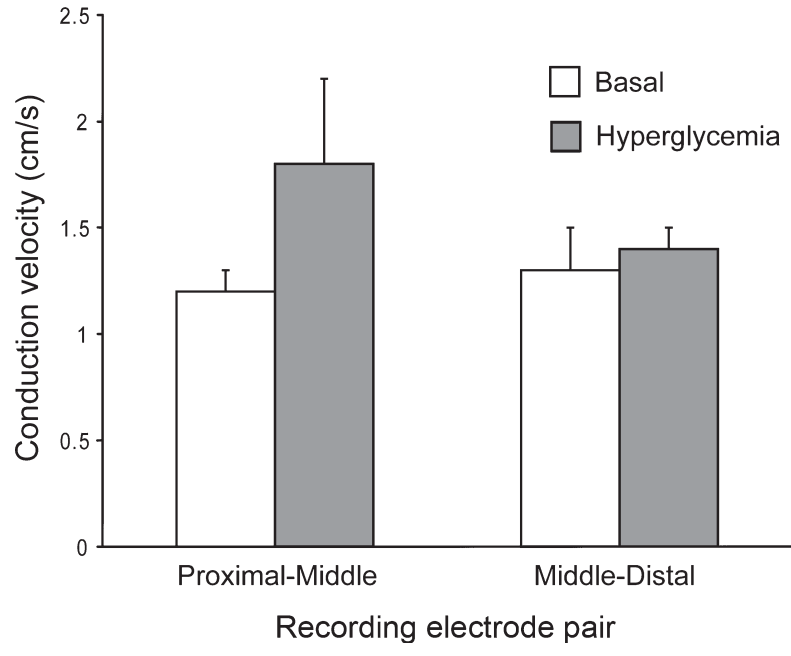

Figure 6 Conduction velocities of normally conducted slow waves are plotted from the proximal to the middle recording site and from the middle to the distal site under the two test conditions. Velocities are similar between both recording electrode pairs under both basal conditions and during hyperglycaemic clamping to $250 \mathrm{mg}$.

reduced coupling of slow waves of normal frequency to $54 \pm 6 \%$ between the proximal and middle leads $(P<0.01$ compared with basal conditions $)$ and $47 \pm 4 \%$ between the middle and distal leads $(P<0.01$ compared with basal conditions) (Fig. 5A). Coupling showed a trend to greater reductions distally during hyperglycaemia than proximally $(P=0.11)$. Per cents of segments with $<50 \%$ coupling increased to $50 \pm 13 \%$ proximally $(P<0.01$ compared to basal conditions) and $59 \pm 9 \%$ distally during acute hyperglycaemia $(P<0.01$ compared with basal conditions $)$ (Fig. 5B). Propagation velocities were unaffected by hyperglycaemia proximally $\left(1.8 \pm 0.4 \mathrm{~cm} \mathrm{~s}^{-1}, P=0.20\right)$ and distally $\left(1.4 \pm 0.1 \mathrm{~cm} \mathrm{~s}^{-1}, P=0.75\right)$ (Fig. 6).

\section{DISCUSSION}

Diabetic gastropathy is postulated to result from several factors related to gastric motor, sensory and myoelectric function. Many patients exhibit delayed solid food emptying, which may be secondary to reduced antral contractions, increased antral diameter or spastic pyloric motor activity. ${ }^{1-5}$ Phasic antral pressure wave activity is regulated by a regular cyclic myoelectric phenomenon, the slow wave. ${ }^{6}$ Under healthy conditions, the slow wave is generated at a frequency of $3 \mathrm{cpm}$ by a pacemaker region in the proximal body and is conducted without interruption in an antegrade direction to the pylorus. ${ }^{17}$ Phasic contractions elicited by meals or neurochemical stimulation result from enhancement of the slow wave plateau potential or by induction of action potentials in phase with the slow wave. ${ }^{18-20}$ Many diabetics with gastroparesis exhibit alterations in slow wave frequency including those with rapid cycling (tachygastria) or with frequency retardation (bradygastria)., ${ }^{7,2,21}$ Others show impaired slow wave amplitude responses 
after eating. ${ }^{21}$ Some diabetics with normal gastric emptying exhibit heightened gastric sensitivity suggestive of afferent dysfunction, but others show similar slow wave abnormalities as those with gastroparesis indicating that some myoelectric disturbances do not grossly disrupt gastric motor activity. ${ }^{3}$

In vivo and in vitro animal studies suggest possible mechanisms by which disrupted slow wave activity may impair motor activity. Tachygastria reduces plateau potential activity thereby rendering the stomach insensitive to contractile stimulation, while bradygastria decreases the maximal contractile frequency during stimulation. ${ }^{18-20}$ Disturbances in slow wave coupling between adjacent regions have been characterized in canine models. ${ }^{15,22}$ Such uncoupling of slow waves of normal frequency promotes ineffective propagation of pressure waves. Uncoupling of dysrhythmic slow waves also occurs with further disruption of motor activity.

Motor and myoelectric disturbances in diabetic gastropathy are influenced by the degree of glycaemic control. Gastric emptying in type I and type II diabetics is delayed during acute hyperglycaemia to levels above $270 \mathrm{mg} \mathrm{dL}^{-1} \cdot{ }^{2,23}$ Likewise, the percentage of recording time in slow wave dysrhythmic activity decreases from $41 \%$ during hyperglycaemia to $6 \%$ during euglycaemia in diabetics with gastropathy. ${ }^{9}$ The ability of acute hyperglycaemia to disrupt gastric function in nondiabetic healthy humans reinforces the contributions of metabolic factors in diabetic gastropathy. Indeed, hyperglycaemia inhibits gastric migrating motor complex activity, blunts the fed motor response, stimulates pyloric activity and elicits tachygastria in healthy volunteers. ${ }^{10,24,25}$

These investigations define slow wave abnormalities in diabetes and in acute hyperglycaemic models of diabetic gastropathy. However, they provide limited insight into patterns of myoelectric dysfunction that may underlie motor disruption. Cutaneous electrogastrography is often employed to detect gastric tachyarrhythmias and abnormalities of slow wave power in diabetic models. ${ }^{7,8,21}$ Multichannel electrogastrography has been proposed as a means of characterizing regional slow wave differences as well as coupling between adjacent regions and propagation velocities. ${ }^{26,27}$ However, all single and multichannel electrogastrography methods provide summations of slow wave activity from large gastric regions and are limited in their abilities to detect isolated dysrhythmias, to measure regional differences in power, and to quantify local coupling and propagation properties. Recently, our laboratory validated a method to map slow wave parameters employing upper endoscopy to direct a bipolar recording probe to different antral regions. ${ }^{12}$ Using this method, regional differences in power under normal conditions and differential dysrhythmic effects of two stimuli (glucagon and acute hyperglycaemia) were characterized. ${ }^{13}$ Because this method measured regional differences in slow wave frequency during a given test condition, it provided an indirect means of detecting uncoupling as slow waves of slightly different frequency by definition cannot be coupled. However, this technique was unable to acquire signals from more than one site at any time. Thus, direct quantification of coupling and propagation direction and velocity was not possible.

In the present study, we employed a novel multichannel recording method that can acquire slow wave signals from several sites simultaneously during basal conditions and hyperglycaemic clamping. Using this technique, the frequency stability and high degree of slow wave coupling between adjacent regions under basal conditions was confirmed and regional power gradients that increased from proximal to distal recording sites were quantified. As electrical activity relates to phasic motor function in the distal stomach, it is likely that the wide variations in signal power distally relate to the more intense contractions that develop in the distal antrum compared with the gastric corpus. The method also provided qualitative and quantitative measures of conduction, including propagation velocity. Dysrhythmic effects of hyperglycaemic clamping to $250 \mathrm{mg} \mathrm{dL}^{-1}$ on slow wave activity were characterized. Tachygastrias were observed with dominant frequencies that ranged from 4 to $8 \mathrm{cpm}$. New findings included the observations that most hyperglycaemia-evoked tachygastrias are localized to the distal antrum and that uncoupling between adjacent sites occurs even when there are no quantifiable slow wave frequency abnormalities. Although most tachygastrias localized to a single recording site, some were observed in tracings from two or three adjacent electrodes. In some instances, these tachygastrias exhibited similar frequencies across adjacent recording sites. However, coupling of adjacent tachygastrias was difficult to calculate due to short dysrhythmia duration and frequency irregularity. In contrast, hyperglycaemia had limited effect on proximal to distal power gradients and no effect on propagation velocities of normally coupled slow waves.

This investigation provides insight into patterns and potential mechanisms of slow wave disruption in models of diabetic gastropathy. Our results mirror those of other investigations that report increased sensitivity of the distal antrum to slow wave disruption in non-diabetic models. ${ }^{28,29}$ In such 
studies, distal antral ectopic pacemaker activity promotes retrograde myoelectric propagation that inhibits normal contractile function. Because most tachygastrias in this investigation were localized to a single site, reliable determination of tachygastric propagation could not be performed. Reduced coupling between adjacent regions during hyperglycaemia represents a plausible mechanism for disrupted contractile propagation. The proximal to distal power gradient may be a consequence of the thicker distal musculature distally. The cause of the increased distal power variability is unknown, but may relate to local neurohumoral factors. The limited effects of hyperglycaemia on the power gradient, power variability and propagation velocities suggest that these parameters may be more resistant to metabolic disruption. However, as this study was performed in healthy humans, its findings may not be definitively extrapolated to patients with diabetes. Studies in diabetic gastropathy are ongoing.

Concerns can be raised about this investigation. The effects of endoscopic air insufflation of the stomach and clipping of electrodes to the mucosa on slow wave parameters are uncertain. However, basal rhythms and dysrhythmic degeneration during hyperglycaemia in this study are similar to those observed with electrogastrography. It is likely the current methods are less disruptive than those of our previous studies, in which an endoscope was left in place during recordings. In that investigation, basal and stimulated slow wave patterns were similar to those of the current study suggesting that the slow wave is relatively resistant to endoscopy-directed recording techniques. As subjects were partially sedated during much of the recordings, symptom correlations with slow wave disruptions were not possible. In previous studies in non-sedated healthy individuals undergoing hyperglycaemic clamping to similar degrees, we have observed minor degrees of fullness and early satiety. ${ }^{10,13}$ The relation of these findings to other gastric functional parameters must be validated. Concurrent manometry could confirm effects of uncoupling or regional dysrhythmias on localized contractile propagation. A limitation of the current method is the wide spacing between recording electrodes that precludes detailed characterization of local propagation patterns that, as in the heart, might include retrograde or re-entrant patterns. In vitro studies employing 240 recording probes provide such careful definition of slow wave activity in excised tissues. ${ }^{30}$

Mucosal mapping techniques may be employed to derive important new information. As stated above, these observations must be correlated with those in diabetics with symptoms of gastropathy or with impaired motor function. In the present study, healthy humans without underlying neuromuscular damage to the stomach were subjected to high plasma glucose levels for $<1 \mathrm{~h}$. It is possible that diabetics might exhibit different or more severe disruptions of slow wave rhythm or coupling that seen in this investigation. However, the near normalization of slow wave rhythm on electrogastrography in diabetics undergoing euglycaemic clamping suggests that the findings of the present study may be relevant to diabetics during periods of poor glycaemic control. ${ }^{9}$ Knowing the propagation patterns of gastric slow wave activity also may have therapeutic importance. Resection of tachygastric foci is reported to relieve symptoms in selected cases of refractory gastroparesis. ${ }^{31}$ Gastric electrical stimulation uses a low energy stimulus delivered at a single point along the greater curvature of the stomach. ${ }^{32,33}$ Next generation neurostimulators and gastric pacemakers are under development that will deliver depolarizing impulses to several sites along the gastric wall. ${ }^{34,35}$ It is unknown if intact conduction will enhance the effectiveness of these new treatments or if uncoupling or dysrhythmias will reduce their therapeutic benefits. If the latter is true, mapping technologies may be useful to assist in placing stimulating electrodes for such therapies. Future studies using more elaborate methods are needed to address these possibilities.

In conclusion, gastric slow wave activity exhibits stable, highly coupled rhythms with an increasing proximal to distal power gradient under basal conditions. Acute hyperglycaemia elicits isolated tachygastrias and uncoupling of normal slow waves that are most prominent in the distal stomach. In contrast, hyperglycaemia has limited effect on power gradients and does not disrupt gradients in power variability or conduction velocities of normally coupled slow wave waves. These findings using multichannel mucosal mapping provide insight into myoelectric disturbances that may underlie the gastric functional abnormalities of diabetic gastropathy and provide a foundation for studying slow wave conduction defects in this condition.

\section{ACKNOWLEDGMENTS AND DISCLOSURES}

Dr Hasler receives grant funding from the NIH (1 U01 DK073985-01). The authors acknowledge the invaluable assistance of the nursing staff of the General Clinical Research Center at the University of Michigan Hospital where all studies were performed. The 
authors have no other relevant disclosures or conflicts of interest.

\section{REFERENCES}

1 Horowitz M, Maddox AF, Wishart JM, Harding PE, Chatterton BE, Shearman DJ. Relationships between oesophageal transit and solid and liquid gastric emptying in diabetes mellitus. Eur J Nuc Med 1991; 18: 229-34.

2 Horowitz M, Harding PE, Maddox AF et al. Gastric and oesophageal emptying in patients with type 2 (non-insulin-dependent) diabetes mellitus. Diabetologia 1989; 32: 151-9.

3 Samsom M, Salet GA, Roelofs JM, Akkermans LM, Vanberge-Henegouwen GP, Smout AJ. Compliance of the proximal stomach and dyspeptic symptoms in patients with type I diabetes mellitus. Dig Dis Sci 1995; 40: 2037-42.

4 Mearin F, Camilleri M, Malagelada J-R. Pyloric dysfunction in diabetics with recurrent nausea and vomiting. Gastroenterology 1986; 90: 1919-25.

5 Camilleri M, Malagelada J-R. Abnormal intestinal motility in diabetics with the gastroparesis syndrome. Eur J Clin Invest 1984; 14: 420-7.

6 Hasler WL, Owyang C. Physiology and pathophysiology of the interstitial cells of Cajal: from bench to bedside. VI. Pathogenesis and therapeutic approaches to human gastric dysrhythmias. Am J Physiol 2002; 283: G8-15.

7 Jebbink HJ, Bruijs PP, Bravenboer B, Akkermans LM, Vanberge-Henegouwen GP, Smout AJ. Gastric myoelectrical activity in patients with type I diabetes mellitus and autonomic neuropathy. Dig Dis Sci 1994; 39: 2376-83.

8 Koch KL, Stern RM, Stewart WR, Vasey MW. Gastric emptying and gastric myoelectrical activity in patients with diabetic gastroparesis: effect of long-term domperidone treatment. Am J Gastroenterol 1989; 84: 1069-75.

9 Jebbink RJ, Samsom M, Bruijs PP et al. Hyperglycemia induces abnormalities of gastric myoelectrical activity in patients with type I diabetes mellitus. Gastroenterology 1994; 107: 1390-7.

10 Hasler WL, Soudah HC, Dulai G, Owyang C. Mediation of hyperglycemia-evoked gastric slow-wave dysrhythmias by endogenous prostaglandins. Gastroenterology 1995; 108: 727-36.

11 Parkman HP, Hasler WL, Barnett JL, Eaker EY. Electrogastrography: a document prepared by the gastric section of the American Motility Society Clinical GI Motility Testing Task Force. Neurogastroenterol Motil 2003; 15: 89-102.

12 Coleski R, Hasler WL. Directed endoscopic mucosal mapping of normal and dysrhythmic gastric slow waves in healthy humans. Neurogastroenterol Motil 2004; 16: 55765.

13 Coleski R, Sherif A, Nojkov B, Hasler W. Divergent effects of different dysrhythmic stimuli on regional gastric slow wave activity in healthy humans as measured by directed endoscopic mapping (abstract). Neurogastroenterol Motil 2003; 15: 36.

14 De Fronzo RA, Tobin JD, Andres R. Glucose clamp technique: a method for quantifying insulin secretion and resistance. Am I Physiol 1979; 237: E214-23.

15 Verhagen MA, Van Schelven LJ, Samsom M, Smout AJ. Pitfalls in the analysis of electrogastrographic recordings. Gastroenterology 1999; 117: 453-60.
16 Mintchev MP, Otto SJ, Bowes KL. Electrogastrography can recognize gastric electrical uncoupling in dogs. Gastroenterology 1997; 112: 2006-11.

17 Bauer AJ, Publicover NG, Sanders KM. Origin and spread of slow wave in canine gastric antral circular muscle. Am J Physiol 1985; 249: G800-6.

18 Morgan KG, Szurszewski JH. Mechanisms of phasic and tonic actions of pentagastrin on canine gastric smooth muscle. I Physiol 1980; 301: 229-42.

19 Publicover NG, Sanders KM. Effects of frequency on the wave form of propagated slow waves in canine gastric antral muscle. J Physiol 1986; 371: 179-89.

20 Sanders KM, Publicover NG. Excitation-contraction coupling in gastric muscles. Dig Dis Sci 1994; 39 (Suppl. 12): 69S-72S

21 Chen JD, Lin Z, Pan J, McCallum RW. Abnormal gastric myoelectrical activity and delayed gastric emptying in patients with symptoms suggestive of gastroparesis. Dig Dis Sci 1996; 41: 1538-45.

22 Mintchev MP, Bowes KL. Do increased electrogastrographic frequencies always correspond to internal tachygastria? Ann Biomed Eng 1997; 25: 1052-8.

23 Fraser RJ, Horowitz M, Maddox AF, Harding PE, Chatterton BE, Dent J. Hyperglycemia slows gastric emptying in type I diabetes mellitus. Diabetologia 1990; 33: 675-80.

24 Barnett JL, Owyang C. Serum glucose concentration as a modulator of interdigestive gastric motility. Gastroenterology 1988; 94: 739-44.

25 Fraser R, Horowitz M, Dent J. Hyperglycaemia stimulates pyloric motility in normal subjects. Gut $1991 ; 32$ : 475-8.

26 Simonian HP, Panganamamula K, Parkman HP et al. Multichannel electrogastrography in normal subjects: a multicenter study. Dig Dis Sci 2004; 49: 594-601.

27 Wang ZS, Elsenbruch S, Orr WC, Chen JD. Detection of gastric slow wave uncoupling from multi-channel electrogastrogram: validations and applications. Neurogastroenterol Motil 2003; 15: 457-65.

28 Qian LW, Pasricha P, Chen JD. Origins and patterns of spontaneous and drug-induced canine gastric myoelectrical dysrhythmia. Dig Dis Sci 2003; 48: 508-15.

29 Sarna SK, Daniel EE. Electrical stimulation of gastric electrical control activity. Am J Physiol 1973; 225: 125-31.

30 Lammers WJ, Stephen B. Origin and propagation of individual slow waves along the intact feline small intestine. Exp Physiol 2008; 93: 334-46.

31 Abell T, McCallum R, Hocking M et al. Gastric electrical stimulation for medically refractory gastroparesis. Gastroenterology 2003; 125: 421-8.

32 Sanders K, Mengut R, Chey W et al. One explanation for human antral tachygastria (abstract). Gastroenterology 1979; 76: 1234.

33 Abell TL, Van Cutsem E, Abrahamsson H et al. Gastric electrical stimulation in intractable symptomatic gastroparesis. Digestion 2002; 66: 204-12.

$34 \mathrm{Xu} \mathrm{J}$, Ross RA, McCallum RW, Chen JD. Two-channel gastric pacing with a novel implantable gastric pacemaker accelerates glucagon-induced delayed gastric emptying in dogs. Am J Surg 2008; 195: 122-9.

35 Mintchev MP, Sanmiguel CP, Amaris M, Bowes KL. Microprocessor-controlled movement of solid gastric content using sequential neural electrical stimulation. Gastroenterology 2000; 118: 258-63. 